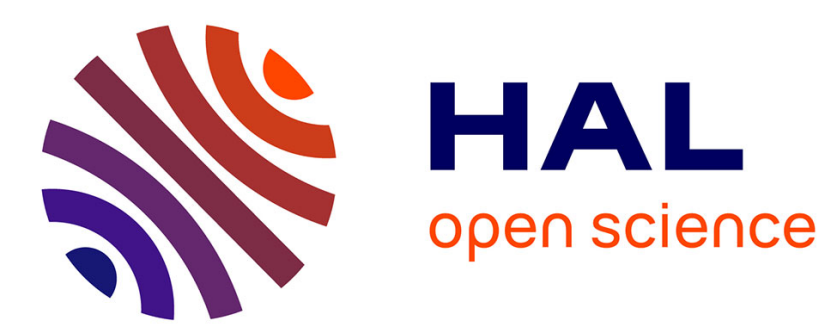

\title{
A non-hydrostatic flat-bottom ocean model entirely based on Fourier expansion
}

\author{
Achim Wirth
}

\section{To cite this version:}

Achim Wirth. A non-hydrostatic flat-bottom ocean model entirely based on Fourier expansion. Ocean Modelling, 2005, 9 (1), pp.71-87. 10.1016/j.ocemod.2004.04.003 . hal-00546305

\section{HAL Id: hal-00546305 \\ https://hal.science/hal-00546305}

Submitted on 19 Nov 2020

HAL is a multi-disciplinary open access archive for the deposit and dissemination of scientific research documents, whether they are published or not. The documents may come from teaching and research institutions in France or abroad, or from public or private research centers.
L'archive ouverte pluridisciplinaire HAL, est destinée au dépôt et à la diffusion de documents scientifiques de niveau recherche, publiés ou non, émanant des établissements d'enseignement et de recherche français ou étrangers, des laboratoires publics ou privés. 


\title{
A non-hydrostatic flat-bottom ocean model entirely based on Fourier expansion
}

\author{
A. Wirth \\ LEGI, BP 53, 38041 Grenoble Cedex 9, France
}

\begin{abstract}
We show how to implement free-slip and no-slip boundary conditions in a three dimensional Boussinesq flat-bottom ocean model based on Fourier expansion. Our method is inspired by the immersed or virtual boundary technique in which the effect of boundaries on the flow field is modeled by a virtual force field. Our method, however, explicitly depletes the velocity on the boundary induced by the pressure, while at the same time respecting the incompressibility of the flow field. Spurious spatial oscillations remain at a negligible level in the simulated flow field when using our technique and no filtering of the flow field is necessary. We furthermore show that by using the method presented here the residual velocities at the boundaries are easily reduced to a negligible value. This stands in contradistinction to previous calculations using the immersed or virtual boundary technique.

The efficiency is demonstrated by simulating a Rayleigh impulsive flow, for which the time evolution of the simulated flow is compared to an analytic solution, and a three dimensional Boussinesq simulation of ocean convection. The second instance is taken form a well studied oceanographic context: A free slip boundary condition is applied on the upper surface, the modeled sea surface, and a no-slip boundary condition to the lower boundary, the modeled ocean floor. Convergence properties of the method are investigated by solving a two dimensional stationary problem at different spatial resolutions.

The work presented here is restricted to a flat ocean floor. Extensions of our method to ocean models with a realistic topography are discussed.
\end{abstract}

E-mail address: achim.wirth@hmg.inpg.fr (A. Wirth). 


\section{Introduction}

Most of the world ocean is known to be in hydrostatic balance at large scales. However, ocean dynamics at scales smaller than about $10 \mathrm{~km}$, convection, circulation in coastal areas, surface mixed layer dynamics, and the dynamics of overflows in straits are instances of ocean dynamics where non-hydrostatic effects are essential. These instances of non-hydrostatic ocean dynamics are important dynamical problems in their own right and also influence the large scale dynamics of the world ocean and the climate system of our planet.

To study the aforementioned instances of non-hydrostatic ocean dynamics numerical models based on the hydrostatic primitive equations are not adapted, and the full three dimensional Navier-Stokes equations have to be implemented instead. The pioneering work by Marshall et al. (1997) is not only an example of this endeavor, but also discusses non-hydrostatic instances in ocean dynamics in detail. The here presented numerical model for the study of non-hydrostatic processes in the ocean is based on Fourier expansions. The virtual boundary technique is used and refined to implement the ocean surface as well as a flat (no-slip) ocean floor. A great variety of non-hydrostatic processes in the ocean do not depend on the topography of the ocean floor and an ocean model specialized to these cases represents a powerful tool in understanding such processes.

Spectral methods based on Fourier expansions are widely used in the simulation of turbulent flow when subject to periodic boundary conditions. For such cases this method is unbeaten in accuracy and speed. This success is based on the pseudo-spectral method Gottlieb and Orszag (1977), where derivatives are performed in Fourier and non-linear operations in physical space, and the fast Fourier transform is used to pass from one space to the other. The efficiency of the pseudo-spectral method resides in the locality of the operations performed in the corresponding spaces and, overall the efficiency of the fast Fourier transform, the sole non-local operation, which only requires $\mathrm{O}(n \log n)$ operations.

Spectral methods based on Fourier expansions in one, two or three directions are widely spread in ocean modeling when specific processes are studied and periodic boundary conditions are present in these directions (Julien et al., 1996; Wirth, 2000). Methods based on Fourier expansions have, so far, not seen the same success when it comes to flows involving boundaries. In such cases Chebyshev polynomials are usually employed in the directions subject to non-periodic boundary conditions (Davies and Lawrence, 1994; Julien et al., 1996). The difficulty lies in the fact that two very different kinds of conditions have to be imposed: First, the condition of zero divergence which is easily imposed in Fourier space, second, the boundary conditions which are imposed in physical space. As both conditions "reside" in different spaces, the challenge lies in imposing them simultaneously. New momentum in surmounting the apparent incompatibility of imposing simultaneously a zero divergence condition and boundary conditions in models based on Fourier expansion came with the introduction of the immersed or virtual boundary method Peskin (1977) (see also Iaccarino and Verzicco (2003) for a recent review). The term immersed is usually used for boundaries that vary in time while virtual boundaries are stationary. As we are here only considering stationary boundaries we will be using the term virtual boundaries.

In their pioneering work on the implementation of virtual boundaries in a model based on Fourier expansion, Goldstein et al. (1993) used a feedback forcing that reduced the velocities at boundary points. The forcing depended on two parameters and introduced considerable stiffness resulting in a very short time step. Their flow showed global spatial oscillations although they 
used a smoothed force field. Such smearing of the force field and thus of the boundary reduces the accuracy of the solution especially near the boundary. Furthermore, a spectral smoothing technique had to be employed to obtain a smooth flow field even for temporally steady flow. An important point of their paper is the finding, that the spurious oscillations can be reduced by adjusting a fictitious flow field inside the solid body.

This technique is explored further in the present paper. We indeed demonstrate a way of applying a force at the boundary and inside the solid body only, resulting in a flow field with no spurious oscillations. Contrary to Goldstein et al. (1993) we do not use a feedback forcing in which the velocity at boundary points is damped towards the required value, but we use the direct forcing method, see Mohd-Yosuf (1997), in which a force field on the boundary insures the boundary conditions. For a detailed discussion of feedback and direct forcing we refer the reader to the review by Iaccarino and Verzicco (2003) and to references therein. By using the direct forcing method rather than a feedback forcing we do not have any time step limitations due to the virtual boundaries and there are no additional parameters to adjust.

In our method the direct forcing is split in two parts, the first correcting the spurious velocity at the boundary due to the advective, buoyancy and diffusive terms and the second accounting for the spurious contributions due to the pressure term. It is the second part that is usually not explicitly accounted for when virtual boundary conditions are used.

In previous calculations using virtual boundaries a residual velocity of $10^{-3}-10^{-4}$ times the maximal velocity was observed at the boundary Iaccarino and Verzicco (2003). For oceanic applications these levels of residual velocity through the boundary are not acceptable. With our method the reduction of the residual error is only subject to limitations due to the machine precision.

In the next section we explain our method. Section 3 discusses the extension of the introduced method to more complex boundaries. The two test cases are exposed and numerical details are explained in Section 4. Concluding remarks appear in Section 5. The important but technical question of convergence properties of the method presented here are discussed in Appendix A.

\section{The method}

The present numerical method deals with simulating the motion of an incompressible Boussinesq fluid in two and three dimensions. The governing equations are

$$
\begin{aligned}
& \partial_{t} \mathbf{u}+\mathbf{u} \cdot \nabla \mathbf{u}+\nabla P=\alpha T \mathbf{e}_{\perp}+v \nabla^{2} \mathbf{u}+\mathbf{F}, \\
& \nabla \cdot \mathbf{u}=0, \\
& \partial_{t} T+\mathbf{u} \cdot \nabla T=\kappa \nabla^{2} T+S,
\end{aligned}
$$

where $\mathbf{u}$ is the velocity field, $T$ the temperature field, $P$ the pressure field and $\alpha$ the thermal expansion coefficient of the fluid. The upward pointing unit vector is denoted by $\mathbf{e}_{\perp}$. The viscosity and diffusivity are given by $v$ and $\kappa$, respectively. The force $\mathbf{F}$ enforces the boundary condition for the velocity and the source term $S$ insures the prescribed heat fluxes through the boundaries. It is clear that the force and the source term have to vanish within the fluid, but not so on the boundary and beyond. 
We restrict ourselves to the case of horizontal upper and lower boundaries. On the upper boundary we apply a free-slip boundary condition, that is a vanishing vertical (normal) velocity component $\left(\mathbf{u} \cdot \mathbf{e}_{\perp}=0\right)$ while there is no direct influence of the boundary on the horizontal velocity component. The lower boundary is subject to a no-slip boundary condition, which means that all components of the velocity vector vanish at the lower boundary $(\mathbf{u}=0)$. We thus model the dynamics in a part of an ocean of constant depth, with a rigid lid, and subject to periodic boundary conditions in the horizontal direction(s).

In the following we will proceed by guiding the reader through one time step of integration with our method. To integrate the Navier-Stokes equations we use the splitting technique (Chorin, 1968; Temam, 1969; Lamb, 1994), where the momentum equation is first solved without considering the pressure term and the thus obtained velocity field is then, in the second part, projected into the space of vector fields with zero divergence.

We start the integration from a velocity field that not only satisfies the boundary conditions, but its components are also symmetric or skew symmetric across the boundary with respect to the vertical direction (normal to the boundary). To this end it is necessary to have a buffer zone above the top and below the bottom boundary as indicated in Fig. 1. More precisely, components that vanish at the boundary as for example, all velocity components at a no slip boundary or the normal velocity at a free slip boundary are continued skew symmetrically beyond the boundary in the buffer zone. For quantities of which the first derivative vanishes, as for example the tangent velocity at a free slip boundary or a scalar like temperature, the boundary value has to be extrapolated from the interior of the flow and the fictitious values inside the solid body (= buffer zone) are continued using even symmetry. The thickness of the buffer zone plays an important role in the implementation due to the non-local nature of spectral representations, and thus the avoidance of spurious oscillations.

We then integrate the momentum equation ignoring contributions from the pressure term

$$
\frac{\tilde{\mathbf{u}}-\mathbf{u}}{\Delta t}=-\mathbf{u} \nabla \mathbf{u}+\alpha T \mathbf{e}_{\perp}+v \nabla^{2} \mathbf{u}+\mathbf{F}_{\mathbf{1}} .
$$

It is easily verified that the even and skew symmetry with respect to the boundary are conserved by the advective and the diffusive term. As a consequence, the resulting velocity field $\tilde{\mathbf{u}}$ verifies the

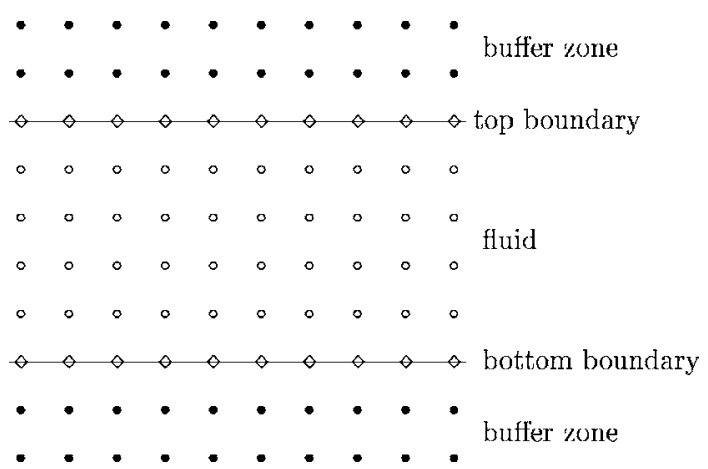

Fig. 1. Decomposition of domain; grid points are marked as open circles for fluid, diamonds for boundary and full circles for buffer points. 
boundary condition except for the discretization error and the error due to the finite thickness of the buffer zone, provided that the buoyancy force $\alpha T$ is zero on the boundary. This means that the force $\mathbf{F}_{1}$, which insures the boundary conditions, is small at the boundary (and zero elsewhere) and will not give rise to spurious oscillations in the fluid flow.

In the next step we orthogonally and linearly project the velocity field $\tilde{\mathbf{u}}$ in the space of divergence free vector fields,

$$
\tilde{\tilde{\mathbf{u}}}=\Pi(\tilde{\mathbf{u}}),
$$

using the projector $\Pi(\mathbf{u})=\nabla^{-2} \nabla(\nabla \cdot \mathbf{u})$, where $\nabla^{-2}$ is the inverse Laplace operator, a well defined operator when applied to functions of vanishing mean (see Peyret, 2002).

Thus the resulting velocity field, $\tilde{\tilde{\mathbf{u}}}$, is of zero divergence, but does not satisfy the boundary conditions. The crucial idea in our method is now to change the velocity field at the boundary (by applying the force $\mathbf{F}_{2}$ that changes $\tilde{\tilde{\mathbf{u}}}$ to $\tilde{\tilde{\mathbf{u}}}+\mathbf{u}_{\pi}$ ) so that after a second projection we obtain

$$
\mathbf{u}=\Pi\left(\tilde{\tilde{\mathbf{u}}}+\mathbf{u}_{\pi}\right) \text {, }
$$

where the final velocity field $\mathbf{u}$ is divergence free and satisfies the boundary conditions. Although the force $\mathbf{F}_{2}$, acting at the boundary only, is the only force having a substantial amplitude, it does not create spurious oscillations within the fluid, as the projection itself is a linear operation which is local in Fourier space. The actual calculation of the force $\mathbf{F}_{\mathbf{2}}$ is the essence of our method and will be given in just a moment.

Let us first put all the parts of our calculation together and we find that our scheme can be written as

$$
\frac{\mathbf{u}^{n+1}-\mathbf{u}^{n}}{\Delta t}=\Pi\left(-\mathbf{u}^{n} \nabla \mathbf{u}^{n}-\alpha T^{n}+v \nabla^{2} \mathbf{u}^{n}+\mathbf{F}_{1}^{n}+\mathbf{F}_{2}^{n}\right) .
$$

In this condensation we used the linearity and idempotents of the projector $\Pi$.

We emphasize once more, that the two forces act only on the boundary or inside the solid body and vanish within the fluid. The crucial question is now of course how to obtain the two forces: The first is actually never calculated but the components of the velocity field are either set to zero at the boundary and continued inside the solid body so that it is skew symmetric along the vertical direction with respect to the boundary, or the boundary value is extrapolated from inside the flow and continued inside the solid body so that it is even symmetric along the normal direction with respect to the boundary, depending on whether the component is to vanish on the boundary or not. Instead of calculating the force $\mathbf{F}_{2}$ we use the equivalent procedure of adding a velocity field $\mathbf{u}_{\pi}$ at the boundary which after the projection exactly cancels the residual velocity at the boundary $(\tilde{\tilde{\mathbf{u}}}(\partial B))$. That is

$$
\tilde{\tilde{\mathbf{u}}}+\Pi\left(\mathbf{u}_{\pi}\right)=0 \quad \text { on } \partial B .
$$

It is important to notice that although $\mathbf{u}_{\pi}$ vanishes in the fluid interior the same thing does not apply for $\Pi\left(\mathbf{u}_{\pi}\right)$. This is an immediate consequence of the projection $\Pi(\cdot)$, being local in Fourier space and therefore non-local in physical space.

To obtain $\mathbf{u}_{\pi}$ we first decompose the residual velocity at the boundary in its normal and tangential components, and denote by $\mathbf{e}_{\perp}$, the unit vector in the direction normal to the boundary. 


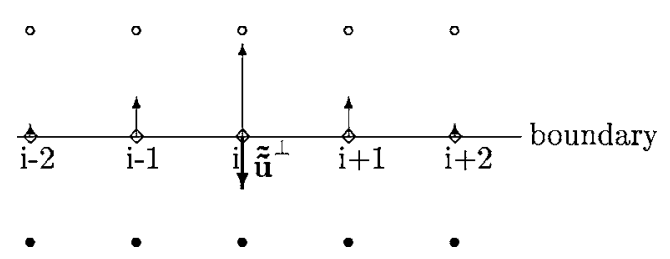

Fig. 2. The thick vector represents the spurious velocity at the boundary $\tilde{\tilde{\mathbf{u}}}(i)$ the thin vectors give the velocity field $\mathbf{u}_{\pi, \mathbf{i}}^{\perp}$, where $\Pi\left(\mathbf{u}_{\pi, i}^{\perp}\right)=-\tilde{\widetilde{\mathbf{u}}}(i)$.

We will start by explaining our method as applied to the normal velocity component in a two dimensional model.

More precisely, if the normal velocity component along the boundary $\left(\tilde{\tilde{\mathbf{u}}}(\partial B) \cdot \mathbf{e}_{\perp}\right)$ vanishes except at the boundary grid-point $i$ (thick vector in Fig. 2), the velocity field $\mathbf{u}_{\pi, i}^{\perp}$ will be non-local along the boundary (thin vectors in Fig. 2). The indices refer to grid points along the boundary, the reference to other directions is omitted for clarity.

As the projection is a linear operation, the vector field $\mathbf{u}_{\pi, i}^{\perp}(j)$ can be calculated in advance with $\Pi\left(\mathbf{u}_{\pi, i}^{\perp}(j)\right)=-\mathbf{e}_{\perp} \delta_{i}(j)$, where the discrete delta function is one at the boundary point $i$ and zero elsewhere.

We then multiply every vector of the vector field by the magnitude of the spurious velocity component at the boundary point $i$ and then sum over all the boundary points. We thus obtain the desired velocity field

$$
\mathbf{u}_{\pi}^{\perp}(j)=\sum_{i}\left(\tilde{\tilde{\mathbf{u}}}(i) \cdot \mathbf{e}_{\perp}\right) \mathbf{u}_{\pi, i}^{\perp}(j) .
$$

In the case of flat boundaries the problem is homogeneous in the horizontal direction and the vector at a location $j$ of the unit vector field $\mathbf{u}_{\pi, i}^{\perp}(j)$ is a function of the distance along the boundary $(i-j)$, only. This means only one unit vector field at the boundary (e.g., $\left.\mathbf{u}_{\pi, 1}^{\perp}\right)$ has to be precalculated. This calculation is performed, before the actual integration of the model in a negligible amount of computation time, the result is stored in a scalar field having the size of the horizontal extension of the model.

The procedure is then repeated for the tangential component(s) of the residual velocities at no-slip boundaries. One additional difficulty however arises in the context of depleting the tangential velocity component(s) with our method. The tangent vector field that vanishes everywhere on the boundary except at one grid point can not be divergence free, and can thus not be the result of the projection operator. This is because at the highest wavenumber resolved in the model (the Nyquist wavenumber) only the cosine and not the sine wave is represented. The problem is however easily resolved by allowing the vector field to possess a divergence at the Nyquist wavenumber for wave-vectors aligned with the boundary. This is not a problem as the velocity field is put to zero on the boundary and is thus trivially divergence free with respect to horizontal wave-vectors. The correction is thus composed of vector-fields having a non-vanishing divergence, but the divergence cancels when these vector-fields are added together. No artificial divergence at the smallest scale is introduced in the model.

An additional problem arises due to the existence of two distinct boundaries. When the velocity correction at the boundary is added and the projection performed, the non-locality of the projection operation results in a (small) violation of the velocity boundary condition at the opposite 
boundary. This problem is solved by calculating the correction velocity field $\mathbf{u}_{\pi, i}$ not only for the boundary where the correction is being performed, but also at the opposite boundary $\mathbf{u}_{\pi, i}^{\text {op }}$. The velocity field $\mathbf{u}_{\pi, i}^{\text {op }}$ does indeed insure, that the velocity at one boundary is not affected by changes performed on the opposite boundary.

In a last step of the integration the fictitious velocity field in the solid body is again constructed by using even symmetry or skew symmetry across the vertical boundary. The integration then proceeds with the next time step.

\section{Non-horizontal boundary}

In the case of an arbitrary boundary with no symmetries all the vector fields on the boundary $\mathbf{u}_{\pi, i}$ ( $i$ ranging over all the boundary points) have to be precalculated and stored for the actual integration. In a two dimensional model having $n_{1} \times n_{2}$ grid points this would require the storage of $2 \times n_{1}^{2}$ scalars for one arbitrary free-slip boundary. In a three dimensional model having $n_{1} \times n_{2} \times n_{3}$ grid points this the storage requirement increases to $2 \times\left(n \times n_{2}\right)^{2}$ scalars. For larger models this is clearly unfeasible.

If, however, the boundary has some additional symmetry, the storage might be highly reduced. Considering the case of a riblet surface: Such surface is homogeneous in one direction and periodic in the other, with a periodic structure repeating itself every $m$ grid points. For such case the storage is reduced to $2 \times m \times n_{1} \times n_{2}$ scalars for one boundary. With typical values of $m \approx 10$, this represents a negligible amount of storage in the overall numerical integration.

The same idea can be applied to any symmetry present in boundaries as for example a cylinder or a sphere submerged in the fluid. In both cases the implementation of our method is almost analogous to the case of a horizontal boundary.

Extensions of the method presented here to a lower boundary (ocean floor) of arbitrary shape are currently under way and will be published elsewhere.

\section{Validation}

To test our method we used several test cases of varying complexity two of which are presented here: The first is the Rayleigh impulsive flow for which the time evolution of the simulated flow can be compared to an analytic solution. The second is a convection experiment from an oceanographic context in two and three dimensions. We will here present the results from our three dimensional calculations. Convection experiments present a very good test case for methods of imposing boundary conditions as the largest velocities reside near those boundaries. We choose a standard numerical convection experiment (see, e.g., Jones and Marshall (1993), PadillaBarbosa and Métais (2000), and Maxworthy and Narimousa (1994) for laboratory experiments) to facilitate comparison to published results.

The calculations are performed using a pseudo-spectral method based on Fourier expansion. The time stepping is done using a third-order low-storage Runge-Kutta scheme, and the size of the time step is subject to the CFL condition, only.

All calculations presented here were performed on a Pentium 4 lap-top computer. 


\subsection{The Rayleigh impulsive flow}

The fluid domain is a rectangular box measuring $1 \mathrm{~m} \times 1 \mathrm{~m} \times(66 / 64) \mathrm{m}$ length. We suppose that our fluid is initially motionless. At a time $t=0$ a flat horizontal plate in the middle of the fluid impulsively starts to move with a speed of $1 \mathrm{~m} / \mathrm{s}$ in the horizontal direction $\mathbf{e}_{1}$. Viscous effects $\left(v=1 \mathrm{~m}^{2} / \mathrm{s}^{2}\right)$ will spread motion over the entire fluid. The equations are non-dimensionalized by dividing length by $1 \mathrm{~m}$, time by $1 \mathrm{~s}$, velocity by $1 \mathrm{~m} / \mathrm{s}$ and viscosity by $1 \mathrm{~m}^{2} / \mathrm{s}^{2}$. As the spreading of motion is governed by a heat equation, an analytic solution of the velocity field can be obtained for every time $t \geqslant 0$ even when subject to boundary conditions (see, e.g., John (1991)).

The calculations are done with a resolution of $16 \times 16$ points in the horizontal directions. The vertical resolution is 44 , grid points within the fluid. The extension of the buffer zones above the upper surface and below the lower surface are 10 grid points.

The comparison of the model calculation to the analytic solution at different times can be seen in Fig. 3, which shows a generally very good agreement. Deviations are only visible for the first panel $(t=0.01)$. The small deviations in the first panel can be explained by two things: (i) the slightly different initial condition (at $t=0$ ) for the model calculation and the analytic solution. The horizontal velocity in the analytic solution starts with a flow being non-vanishing only at one horizontal level of vanishing thickness, whereas the model starts form the discretized equivalent; (ii) the extrapolation method to obtain the boundary value at a free-slip boundary is of only second order (could easily be extended to higher order).
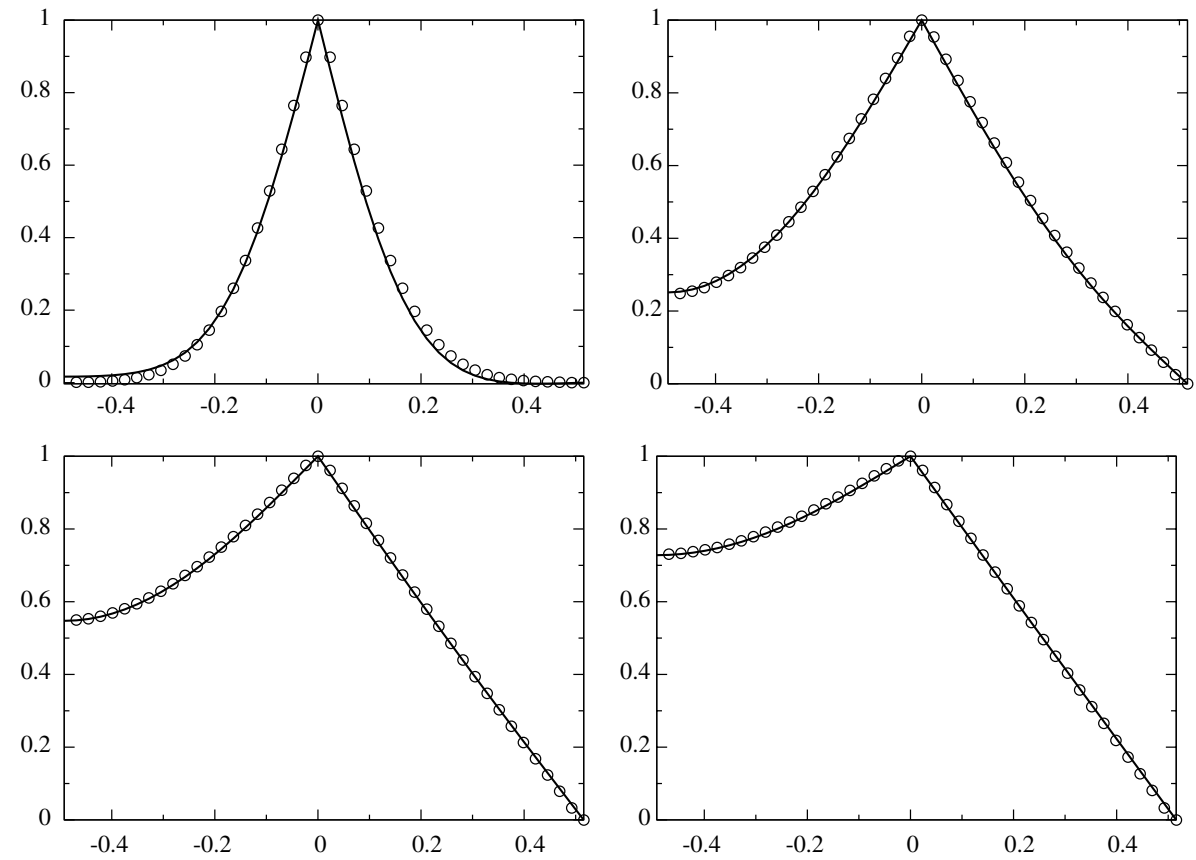

Fig. 3. Comparison of the horizontal velocity at the 44 vertical grid points (circles) to the analytical solution (line) at times 0.01 (upper left), 0.05 (upper right), 0.1 (lower left), 0.15 (lower right). Horizontal axis in the plot corresponds to vertical extension in the model, vertical axis in the plot gives the non-dimensional velocity. 
In this test case the pressure vanishes everywhere and the velocity is always parallel to the boundaries. We have thus not tested the pressure correction, the key part of our method. The pressure correction will however be the key in the next test case.

\subsection{Convection}

In this test case we simulate the sinking of water cooled at the surface in a homogeneous ocean. The simulation is based on the non-hydrostatic Boussinesq equations in a rotating frame and has a free-slip boundary at the top and a no-slip boundary at the bottom. The simulated domain extends $32 \mathrm{~km}$ in both horizontal directions and $2 \mathrm{~km}$ in the vertical. A cooling of $Q_{0}=800 \mathrm{~W} / \mathrm{m}^{2}$ is applied at a perfectly mixed surface layer from zero to $211 \mathrm{~m}$ depth, in a circular horizontal area of radius $R=8 \mathrm{~km}$, outside this disk the heating is rapidly reduced to zero (heating outside the disk $\left.r \geqslant R: Q(r)=Q_{0} \exp \left(1-r^{4} / R^{4}\right)\right)$. The cooling lasts for $48 \mathrm{~h}$. The specific heat capacity of water is $C_{\mathrm{w}}=3900 \mathrm{~J} /(\mathrm{kg} \mathrm{K})$, the constant thermal expansion coefficient is $\epsilon=2 \times 10^{-4} \mathrm{~K}^{-1}$ and the density is $\rho=1000 \mathrm{~kg} / \mathrm{m}^{3}$. The Coriolis parameter is given by $f=1.0 \times 10^{-4} \mathrm{~s}^{-1}$. These values give the buoyancy flux

$$
B_{0}=\frac{\epsilon Q_{0} g}{C_{\mathrm{w}} \rho}=4.025 \times 10^{-7} \mathrm{~m}^{2} / \mathrm{s}^{3} .
$$

Following the scaling arguments of Maxworthy and Narimousa (1994) we get $u_{\text {rot }}=$ $\left(B_{0} / f\right)^{1 / 2}=0.063 \mathrm{~m} / \mathrm{s}$.

The physical model is inspired by observations Schott et al. (1977) and Schott and Leaman (1991), the experimental set up of Maxworthy and Narimousa (1994), and almost identical to the reference experiment of Jones and Marshall (1993) and experiment H4 in Padilla-Barbosa and Métais (2000) to facilitate comparison. One difference of our model to the two models cited above is, that we used a no-slip boundary at the bottom while they used free-slip. We refer the reader to the above mentioned references for a thorough discussion of the physical processes involved.

We add a small amount $\left(\sigma=55 \mathrm{~W} / \mathrm{m}^{2}\right)$ of white-in-space noise to the surface cooling. The horizontal resolution is $128 \times 128$ grid points and there are 44 grid points within the fluid in the vertical direction. Ten grid points above and below the fluid form the buffer zones. The time step, subject to the CFL condition, is $150 \mathrm{~s}$.

The maximal error allowed for the residual normal velocity at the boundaries is smaller than $10^{-10}$ times the maximal velocity in the fluid. At the no-slip boundary the residual tangential velocity is smaller than $10^{-3}$ times the maximal velocity in the fluid. The reduction of the tangential velocity at the lower boundary, using our method, creates a small normal velocity at the upper boundary, and vice versa. Instead of correcting for this contributions explicitly, as it is done for the normal velocities at the lower and upper boundary (see end of Section 2), we choose to iterate the projection and thus reduce such error. The number of iterations needed per step is 3 , for the aforementioned error limits.

By performing several experiments we found the onset of convection being very sensitive not only to the amount and nature of noise added, but also to how the heating at the rim of the disk is reduced to zero. It is also worth mentioning that large eddy simulations were performed in Padilla-Barbosa and Métais (2000) which results in much larger Reynolds and Peclet numbers especially at the onset of the experiment. We thus choose to validate our numerical method to 
compare to the aforementioned published results at the end of the heating period, that is at $t=$ $48 \mathrm{~h}$.

At $t=48 \mathrm{~h}$ and $z=1 \mathrm{~km}$ the vertical velocity ranges from $w_{\min }=-0.14$ to $w_{\max }=0.11 \mathrm{~m} / \mathrm{s}$ (see Fig. 4), this values are close to the results from Jones and Marshall (1993) $\left(w_{\min }=-0.18\right.$ to $w_{\max }=0.09 \mathrm{~m} / \mathrm{s}$, see their Fig. 7d) and to the results from Padilla-Barbosa and Métais (2000), see their Fig. 3. The horizontal scale and structure of the plumes also compare very well in both figures. The temperature isosurface for $T=6.0 \times 10^{-3} \mathrm{~K}$ at $t=48 \mathrm{~h}$ in Fig. 5 are in qualitative and quantitative agreement with those of Padilla-Barbosa and Métais (2000), see their Fig. 2c and $\mathrm{f}$ and those of Jones and Marshall (1993), see their Fig. 5.

In Fig. 6 the three velocity components in the middle of the convective disk at all vertical levels are given. All velocity components are comparable to $u_{\text {rot }}$. The horizontal r.m.s. velocity (averaged over the convective disk) compares perfectly to the results by Jones and Marshall (1993) (see their Fig. 6a and b) for the upper half of the domain. Deviations in the lower half are due to the different boundary condition in our model at the lower boundary. A detailed study of the horizontal r.m.s. velocity (averaged over the convective disk) shows spurious oscillations of small amplitude originating from the lower boundary (no-slip). Please note that the Ekman layer $\left(\Delta_{\mathrm{Ekman}}=32 \mathrm{~m}\right)$ is not resolved in our model $(\Delta z=45 \mathrm{~m})$. Such problem can be avoided when the vertical resolution is increased or a large eddy simulation scheme is used which increases the friction within boundary layers. These spurious oscillations are hidden by the natural variability when the horizontal velocity components are considered (see Fig. 6). They however appear when the natural variability is averaged out. The vertical velocity component is free of such spurious oscillations. This problem is avoided in Jones and Marshall (1993), and Padilla-Barbosa and Métais (2000) by using a free-slip boundary at the bottom.

In all comparisons performed, not all of which are mentioned here, a good overall agreement with the results of Jones and Marshall (1993), and Padilla-Barbosa and Métais (2000) was found.

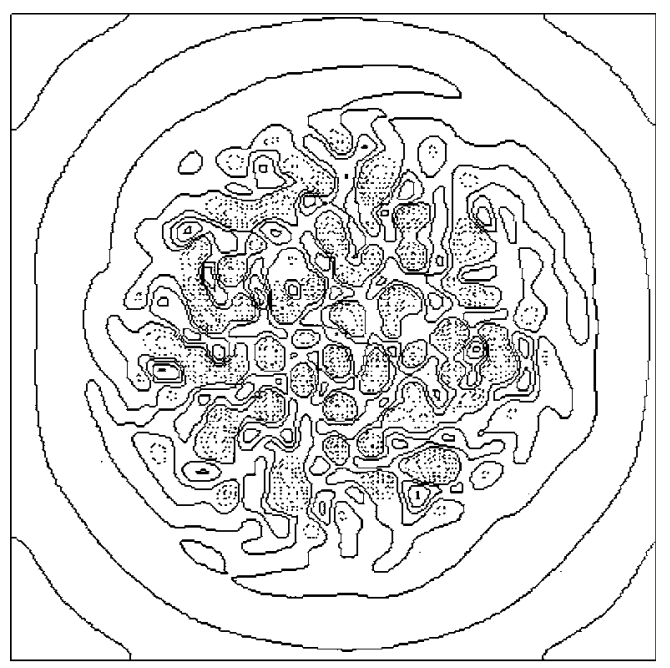

Fig. 4. Vertical velocity at $t=48 \mathrm{~h}$ and depth of $1 \mathrm{~km}$. Contours are drawn every $0.02 \mathrm{~m} / \mathrm{s}\left(w_{\min }=-0.14 \mathrm{~m} / \mathrm{s}\right.$ maximum value is $w_{\max }=0.10 \mathrm{~m} / \mathrm{s}$ ). Dashed lines correspond to negative values (downward velocity). 

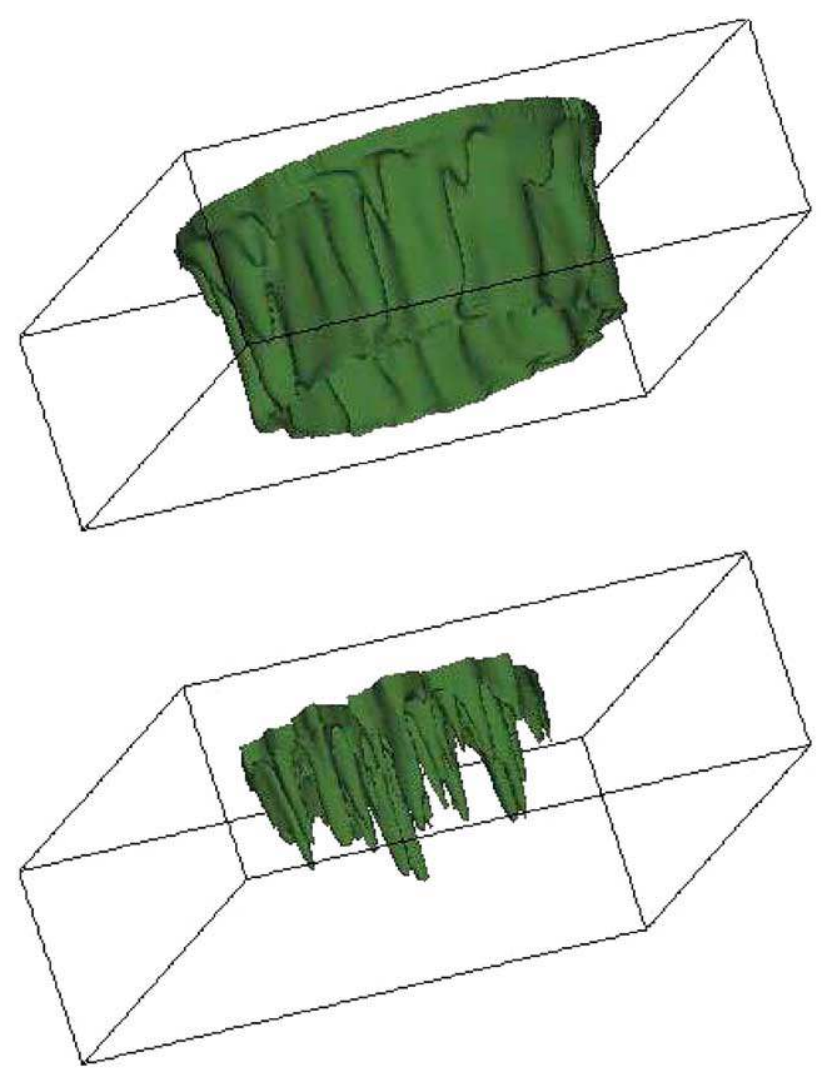

Fig. 5. Temperature isosurface at $t=48 \mathrm{~h}$ for $\Delta T=0.006 \mathrm{~K}$ (top) and $\Delta T=0.03 \mathrm{~K}$ (bottom).

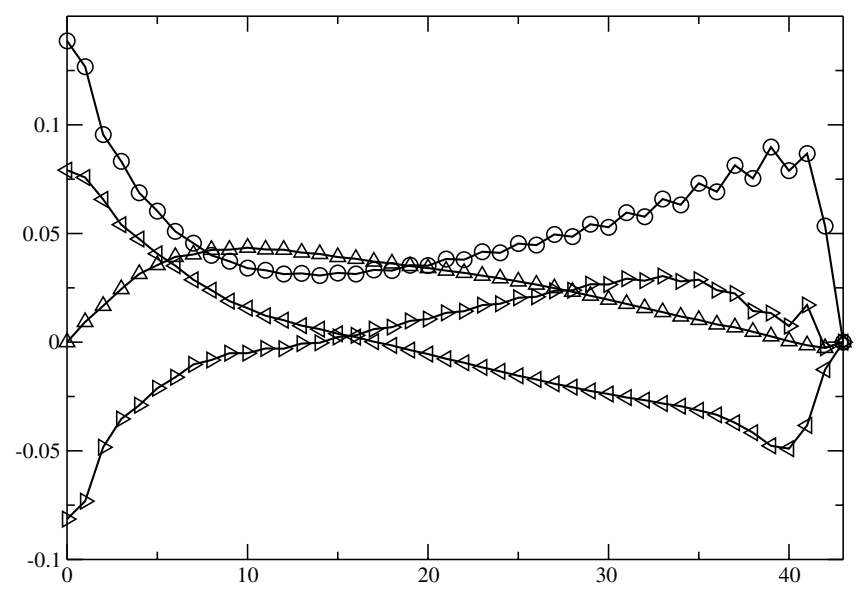

Fig. 6. Horizontal r.m.s. velocity (averaged over the convective disk) at the 44 depth levels (circles). Vertical velocity (triangles up), horizontal velocity $u_{1}$ (triangles left), horizontal velocity $u_{2}$ (triangles right), in the center of the convective disk at the 44 depth levels. All data at $t=48 \mathrm{~h}$. Horizontal axis in the plot corresponds to vertical extension in the model (measured in grip points), vertical axis in the plot gives the velocity in $\mathrm{m} / \mathrm{s}$. 
When the results from both publications differed, our results were closer to those from Jones and Marshall (1993). A finding comes at no surprise as we used their values for the friction and dissipation parameters and we did not use the more sophisticated large eddy simulation scheme of Padilla-Barbosa and Métais (2000) to parameterize the small scales.

\section{Conclusions}

We have introduced a new method of implementing a free-slip rigid-lid and a flat-bottom noslip boundary conditions in ocean models based on Fourier expansion (in all spatial directions). The method is inspired by the immersed or virtual boundary technique. Our method, however, explicitly depletes the velocity on the boundary induced by the pressure while at the same time respecting the incompressibility of the flow field. Using our method the reduction of spurious velocities at the boundaries is only constrained by the computer precision. No constraints, other than CFL, on the time step due to imposing boundary conditions appear. No filtering of the velocity field is necessary and spurious oscillations remain at a negligible level.

The method was tested in various flow configurations involving horizontal boundaries, three of which were shown here. The method is not restricted to horizontal boundaries. Extensions of our method to ocean models with a realistic topography are discussed and further research on this matter is currently under way.

We conclude by reminding the reader that the power of pseudo-spectral method based on Fourier expansion lies not only in their convergence properties but even more in the fact, that the number of operations needed for one time-step is $\mathrm{O}(n \log (n))$, where $n$ is the number of degrees of freedom in the problem. An increase of computer power over time, thus, continually increases the advantage of models based on Fourier expansion.

\section{Acknowledgements}

I am grateful to A. Lanotte, B. Barnier, Y. Morel, K. Schneider and the participants of the "St. Pierre de Chartreuse" workshop for discussion. Help from P. Begou with using the visualisation software is highly appreciated. The work was funded by EPSHOM-UJF No. 00.87.070.00.470.29.25.

\section{Appendix A. Convergence properties}

In this appendix, we explore the convergence properties of the spatial discretization by numerical experiment. To this end errors due to the temporal discretization, being third order in time, have to be reduced. We thus consider a spin-up integration towards a stationary state. The same problem has then to be integrated for different spatial resolutions using the same time-step. To insure the feasibility of such endeavour a simple but challenging test problem has to be chosen. The problem chosen is two dimensional, that is a vertical slice through the ocean that spans 1000 $\mathrm{m}$ in the horizontal and the vertical direction. The sea surface is modeled by a free-slip and the 
ocean floor by a no-slip boundary condition. The boundary condition in the horizontal direction is periodic. In the middle of the domain a vertical (downward) force, with a circular Gaussian profile, is added $\left(G\left(x_{1}, x_{2}\right)=\exp \left(-32 \times 10^{-6} \mathrm{~m}^{-2}\left(x_{1}^{2}+x_{2}^{2}\right)\right)\right)$. Such flow leads to a well studied instance of a non-linear boundary layer at the lower boundary, the so called Hiemenz flow (see e.g. Schlichting, 1968, pp. 96-99). Seven experiments are performed with varying spatial resolution. The number of grid points in the domain (including boundary points) are $8 \times 8,16 \times 16$, $32 \times 32,64 \times 64,128 \times 128,256 \times 256,512 \times 512$. The buffer zone above and below the fluid area is $250 \mathrm{~m}$, each. The friction parameters are $v=\kappa=1.0 \mathrm{~m}^{2} / \mathrm{s}$ and the time-step is $\Delta t=60 \mathrm{~s}$ in all calculations.

The integration started from a vanishing velocity field and was performed for $t_{\max }=2.16 \times 10^{5} \mathrm{~s}$. For the $256 \times 256$ resolution run the integration was continued until $t_{\text {long }}=3.00 \times 10^{5} \mathrm{~s}$. Almost no differences are visible between the velocity fields at $t_{\max }$ and $t_{\text {long }}$ (see Figs. 8-10).

A contour plot of the horizontal velocity component for the lowest and the highest resolution run is given in Fig. 7.

At the lower boundary a Hiemenz flow is generated, and the boundary-layer thickness is about $200 \mathrm{~m}$. We like to mention that in order to capture the non-linear dynamics in the (non-linear) boundary layer about 10 grid points have to be within the boundary layer.

The horizontal velocity along $x=250 \mathrm{~m}$ and the vertical velocity along $x=500 \mathrm{~m}$, as a function of depth are shown in Fig. 8.

The horizontal velocity component clearly exhibits Gibbs oscillations at the lower (no-slip) boundary, the amplitude of the oscillation however decreases linearly with resolution. These oscillations are exposed in Fig. 9.

A linear decrease of the amplitude in the Gibbs oscillations reveals a discontinuity in the first derivative at the boundary. This comes at no surprise as the horizontal velocity component is extended skew symmetrically across the lower boundary. This forces the second derivative to vanish at the boundary which is inconsistent to the real dynamics (see Schlichting, 1968, pp. 9699). No such oscillation occur in the vertical velocity component at the lower boundary (Fig. 10), as a vanishing second derivative is consistent with the real dynamics (see Schlichting, 1968, pp. 96-99). Gibbs oscillations are absent for both velocity components at the upper (free-slip) boundary (Fig. 8).
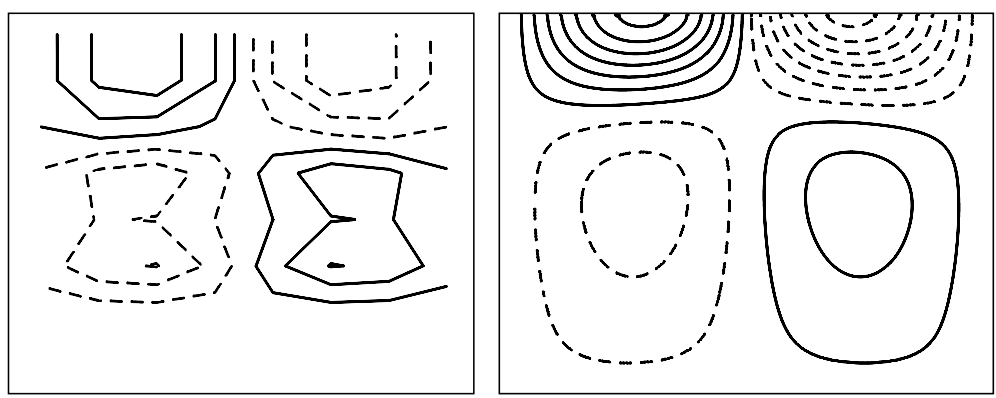

Fig. 7. Contour plot of the horizontal velocity, in the lowest resolution run $(8 \times 8)$ (left) and the highest resolution run $(512 \times 512)$ (right). Contour intervals are drawn every $0.005 \mathrm{~m} / \mathrm{s}$, starting from $-0.0375 \mathrm{~m} / \mathrm{s}$, dashed lines show negative values. 

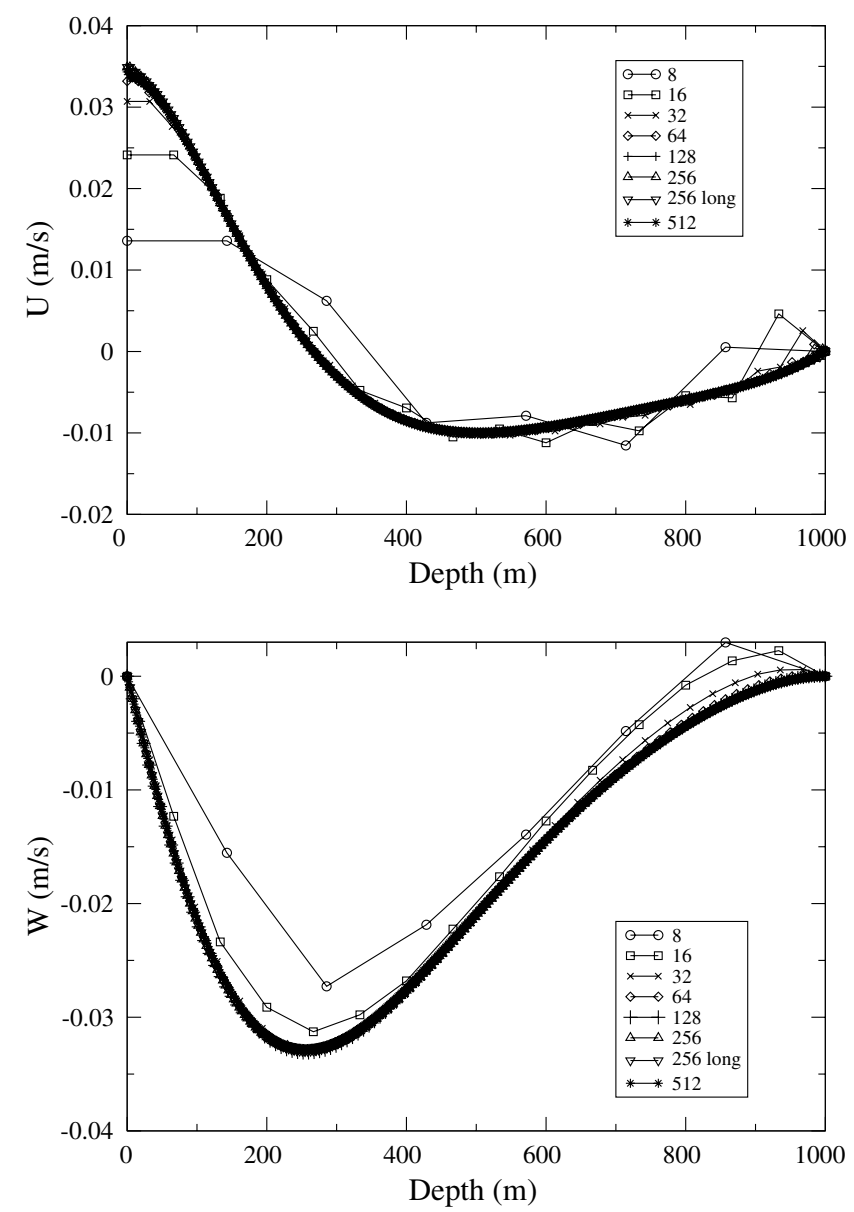

Fig. 8. Horizontal velocity along $x=250 \mathrm{~m}$ (top) and the vertical velocity along $x=500 \mathrm{~m}$ (bottom), as a function of depth. Symbols correspond to the different spatial resolution (as given in the figures).

In Fig. 11 we plot the absolute value of the difference between the highest resolution run and every other run for different variables as a function of the resolution. This difference will be called the "error", we thus chose the highest resolution run as the reference case.

The log-log plot reveals the linear decrease of the amplitude of the Gibbs oscillation of the horizontal velocity component at the lower boundary, already mentioned above. At a fixed distance from the boundary the error however decreases quadratically with the resolution, a behavior typical for Gibbs oscillations. The error in the other quantities measured also points towards the same quadratic decrease of the error with resolution. This decrease is however masked by inaccuracies stemming from the time stepping scheme. For the highest resolution the vertical velocity reaches the CFL-limit (the time step is the same for all the integrations performed), and the error increases for the minimum value of the vertical velocity along $x=500 \mathrm{~m}$ for the high resolution runs. 


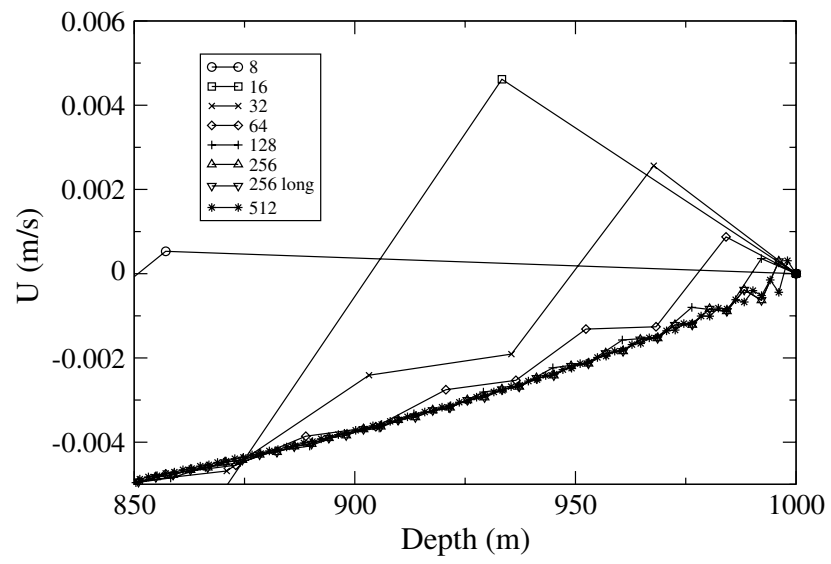

Fig. 9. Horizontal velocity along $x=250 \mathrm{~m}$ near the lower boundary. Symbols correspond to the different spatial resolution (as given in the figure).

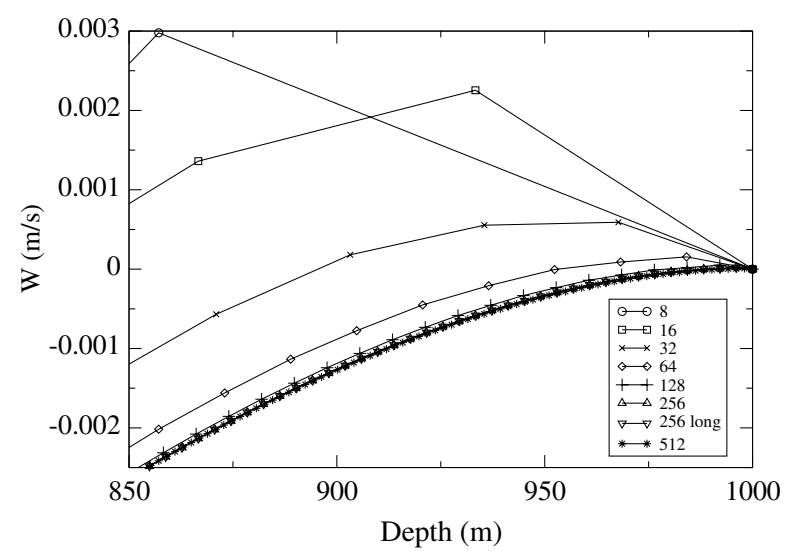

Fig. 10. Vertical velocity along $x=500 \mathrm{~m}$ near the lower boundary. Symbols correspond to the different spatial resolution (as given in the figure).

No spectral convergence is observed in this test-case. This comes at no surprise as the dynamics even in the interior of the domain is completely slaved to the dynamics at the boundaries and thus shows the corresponding convergence properties. It is indeed clearly visible from Fig. 7 that no structures smaller than the basin scale appear in the problem. A problem involving scales smaller than those imposed by the boundaries would cease to be stationary and the here presented analysis could not be performed.

With the insight obtained from boundary-layer theory, the velocity components in the buffer zone can be adjusted so that the real dynamics at the boundary is modeled to higher order. The convergence properties of the dynamics in the interior fluid would then follow. This is the subject of future research. 


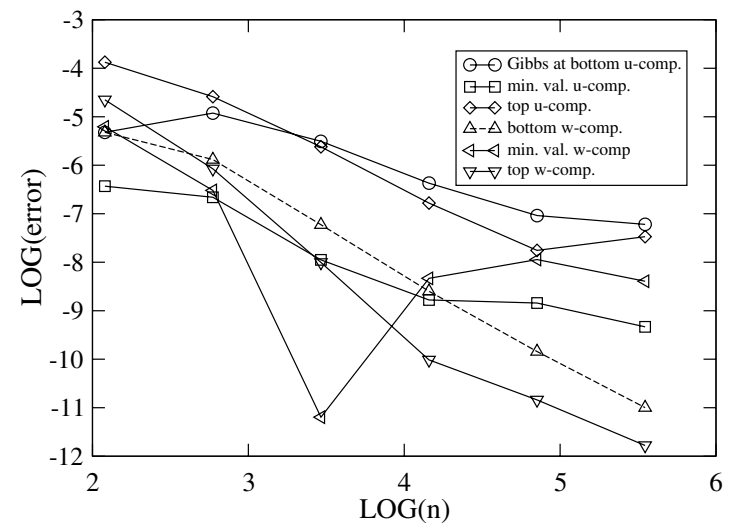

Fig. 11. Log-log plot of the error for different variables: amplitude of the Gibbs oscillation in the horizontal velocity component along $x=250 \mathrm{~m}$ at the lower boundary (circles), minimum value of the horizontal velocity component along $x=250 \mathrm{~m}$ (squares), horizontal velocity component at $x=250 \mathrm{~m}, y=125 \mathrm{~m}$ (diamonds), vertical velocity component at $x=500 \mathrm{~m}, y=875 \mathrm{~m}$ (upward triangles), minimum value of the vertical velocity component along $x=250 \mathrm{~m}$ (leftward triangles) vertical velocity component at $x=500 \mathrm{~m}, y=175 \mathrm{~m}$ (downward triangles).

\section{References}

Chorin, J.A., 1968. Numerical solution of the Navier-Stokes equations. Math. Comput. 22, 745-762.

Davies, A.M., Lawrence, J., 1994. Examining the influence of wind and wind wave turbulence on tidal currents, using a three-dimensional hydrodynamic model including wave current interaction. J. Phys. Oceanogr. 24, 2441-2460.

Goldstein, D., Handler, R., Sirovich, L., 1993. Modeling a no-slip flow boundary with an external force field. J. Comp. Phys. 105, 354-366.

Gottlieb, D., Orszag, S., 1977. Numerical Analysis of Spectral Methods: Theory and Applications. SIAM, Philadelphia. Iaccarino, G., Verzicco, R., 2003. Immersed boundary technique for LES/RANS simulations. Applied Mech. Rev., ASME, in press.

John, F., 1991. Partial Differential Equations, fourth ed. Springer-Verlag, New York.

Jones, H., Marshall, J., 1993. Convection with rotation in a neutral ocean: a study of open-ocean deep convection. J. Phys. Oceanogr. 23, 1009-1039.

Julien, K., Legg, S., McWilliams, J., Werne, J., 1996. Rapidly rotating Rayleigh-Bénard convection. J. Fluid Mech. $322,243-273$.

Lamb, K.G., 1994. Numerical experiments of internal wave generation by strong tidal flow across a finite amplitude bank edge. J. Geophys. Res. 99, 843-864.

Marshall, J., Hill, C., Perelman, L., Adcroft, A., 1997. Hydrostatic, quasi-hydrostatic, and nonhydrostatic ocean modeling. J. Phys. Oceanogr. 27, 5733-5752.

Maxworthy, T., Narimousa, S., 1994. Unsteady, turbulent convection into a homogeneous, rotating fluid, with oceanographic applications. J. Phys. Oceanogr. 24, 865-887.

Mohd-Yosuf, J., 1997. Combined immersed boundary/B-spline methods for simulation in complex geometries. Annual Research Briefs, Center for Turbulence Research, pp. 317-328.

Padilla-Barbosa, J., Métais, O., 2000. Large-eddy simulations of deep-ocean convection: analysis of the vorticity dynamics. J. Turbul., 1009.

Peskin, C.S., 1977. Flow patterns around heart valves: a numerical method. J. Comp. Phys. 25, 220-252.

Peyret, R., 2002. Spectral Methods with Application to Incompressible Viscous Flow. Springer-Verlag, New York. 432 pp.

Schlichting, H., 1968. Boundary-Layer Theory. McGraw-Hill, New York. 817 pp. 
Schott, F., Leaman, K.D., 1991. Observation with moored acoustic Doppler current profiles in the convection regime in the Golf de Lion. J. Phys. Oceanogr. 21, 558-574.

Schott, F., Visbeck, M., Fischer, J., 1977. Observations of vertical currents and convection in the Central Greenland Sea during the winter of 1988-1989. J. Geophys. Res. 98, 14401-14421.

Temam, R., 1969. Sur l'approximation de la solution des équations de Navier-Stokes par la méthode des pas fractionaires II. Archiv. Rat. Mech. Anal. 32, 377-385.

Wirth, A., 2000. The parameterization of baroclinic instability in a simple model. J. Mar. Res. 58, 571-583. 